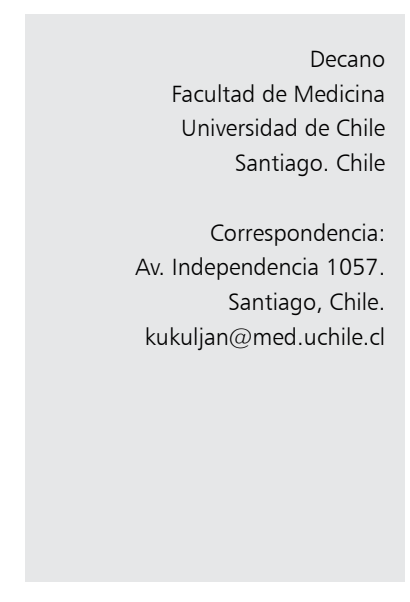

\section{La oportunidad de desarrollar hospitales de alta calidad asistencial y docente para Chile}

\author{
MANUEL KUKULJAN
}

E 1 Ministerio de Salud (MINSAL) está conduciendo un proceso de ordenamiento de la relación entre los recintos asistenciales públicos y las universidades que ofrecen carreras profesionales de la salud. Este ordenamiento, llamado "reasignación de campos clínicos" es una propuesta esperada y que debiera corregir situaciones que no son consistentes con la calidad en salud y educación. De acuerdo con esta iniciativa, los hospitales públicos deberán aplicar una pauta elaborada por el MINSAL para decidir las universidades que podrán desarrollar su actividad en ellos.

Algunos elementos de la propuesta del MINSAL parecen muy adecuados, tales como la consideración de la calidad de las instituciones de educación a partir de su estado de acreditación, la eliminación de las transacciones explícitas que podían determinar el acceso a un campo clínico al mejor postor en términos monetarios, y la declaración de la consideración del valor público de las propuestas de las distintas universidades que aspiran a desarrollar su actividad en un determinado hospital.

Sin embargo, la propuesta adolece de una falta de comprensión profunda de la educación profesional en salud, particularmente de la educación médica, y se queda en el intento de solución de situaciones actuales, sin tomar la oportunidad de mejorar significativamente la calidad de sus hospitales, de aplicar la experiencia nacional e internacional al respecto y de sentar mejores bases para el desarrollo de la salud y la educación en salud para todo el país para las próximas décadas.

\section{Por qué es necesaria la integración entre los procesos asistenciales y de educación}

En las siguientes líneas abordaré el tema central de la integración (que es buena) versus la fragmentación (que es inconveniente) en distintas dimensiones, enfatizando los rasgos esenciales de los mejores hospitales, los que deberíamos emular.

La educación médica se desarrolla principalmente en ambientes asistenciales en los cuales los procesos de atención a pacientes y los de aprendizaje se entrelazan completamente. Existe evidencia sólida de que así se sustenta tanto una mejor calidad de la atención como una mejor calidad de la educación. A modo de ejemplo, los mejores hospitales de Estados Unidos de América, medidos por indicadores de calidad asistencial, son hospitales académicos ${ }^{1,2}$; más aún, el ranking de los mejores hospitales de todo el mundo evidencia la misma situación ${ }^{3}$.

Una característica común a los mejores hospitales es la continuidad e integración entre los procesos asistenciales y de educación, de modo que la mayor parte de los profesionales asistenciales realiza tanto funciones clínicas y docentes; por su parte los estudiantes participan de los procesos de atención conforme a las competencias definidas para cada nivel y bajo la adecuada supervisión (los "estudiantes" comprende desde estudiantes de pregrado a los médicos especialistas en formación avanzada). Otro rasgo fundamental es la integración de la asistencia y la docencia en un único contexto institucional que permite la comunicación, coordinación y conformación 
de equipos indispensables para una atención y una educación de calidad. Adicionalmente, el desarrollo de investigación contribuye de manera determinante a la calidad de los mejores hospitales del mundo.

\section{La participación de los profesionales asistenciales en la docencia es fundamental}

La experiencia y la evidencia al respecto no son exclusivas de países de altos ingresos: modelos comparables se desarrollaron en Chile desde el siglo XIX y con mayor fuerza durante el siglo XX, con la asociación como partes del mismo Estado de hospitales públicos y de la Facultad de Medicina de la Universidad de Chile en Santiago, así como en el Hospital Clínico de la Universidad de Chile, en el Hospital Clínico de la Pontifica Universidad Católica y en centros regionales como Valparaíso, Concepción, Temuco y Valdivia. El éxito de tales modelos es evidente al constatar que la enorme mayoría de los especialistas que trabaja en el sector público y en el sector privado se ha formado y se sigue formando en un número limitado de centros que se desarrolló de este modo, centros que son preferidos por los profesionales en etapa de formación, y cuya impronta es reconocida por pacientes, estudiantes, empleadores y por los procesos de acreditación. Chile debe aspirar a recuperar y reforzar dichos modelos, y tiene los recursos y posibilidades para hacerlo mediante un adecuado diseño y aplicación del ordenamiento que impulsa el Ministerio de Salud.

La propuesta del MINSAL acepta la actual fragmentación de los hospitales en múltiples dimensiones, desde la fragmentación en servicios, sectores e incluso en camas de un servicio en los cuales podrían funcionar distintas universidades, lo que disgrega elementos centrales de la comunicación y coordinación y por cierto atenta contra la calidad en la atención de los pacientes. También se posibilita la fragmentación en niveles, dándose la posibilidad de que los estudiantes de pregrado de una universidad se sobrepongan con profesionales en formación de especialistas de otra universidad, situación impensable en cualquier institución de educación seria, en la que los sistemas, procesos y recursos están integrados.

La fragmentación más profunda y radical que la propuesta perpetúa es aquella entre profesio- nales asistenciales, contratados por los hospitales, y profesionales exclusivamente docentes, pagados por las universidades, visión que destruye un rasgo esencial de la educación médica. Esta división parece sustentarse en la idea de que "a los funcionarios de un hospital se les paga por atender pacientes y no por enseñar", concepción antagónica con la esencia de un buen hospital y que demuestra un significativo desconocimiento de la educación médica, de la historia propia y de los referentes internacionales. Esta visión también es profundamente anómala en lo que respecta a las universidades estatales, porque fragmenta al Estado, que debe actuar articuladamente, en entidades aisladas que deben relacionarse entre sí con reglas del trato con privados, en circunstancias de que el empleador es el mismo Estado y el propósito de contribución al bien público es único y compartido. Esta manera de entender la educación médica implícita en la propuesta es incompatible con la calidad y es un elemento que retrasa y revierte el progreso relativo que experimentó el país durante el siglo XX.

\section{Una oportunidad para generar un bien público} fundamental para el futuro de la salud en Chile

A partir de lo expuesto, creemos que el ordenamiento propuesto debe considerar los sistemas de salud y particularmente los hospitales como entidades integrales y complejas, no divisibles en servicios asignados a instituciones diversas, a menos que existan programas formales y explícitos de colaboración entre universidades y que abarquen en su conjunto todos los niveles y ámbitos de la educación profesional en salud.

La participación de los profesionales asistenciales en la docencia en todos los niveles posibles constituye un elemento central para la calidad de un hospital, por múltiples factores. Los hospitales públicos que definan esta vocación de calidad deben implementar los mecanismos para resguardar este modelo, que por lo demás es el modelo en el cual la vasta mayoría de los más destacados profesionales del país se ha formado antes de la progresiva fragmentación que observamos. Las entidades contraloras, y si es necesario las leyes y reglamentos pertinentes, deben adecuarse para facilitar la generación de un bien público de la mayor trascendencia. La universidades por su 
parte deben proveer los recursos profesionales y académicos que complementan la actividad asistencial, así como los medios para el perfeccionamiento continuo, el reconocimiento adecuado, la actualización y el desarrollo profesional en salud y en educación de todos los miembros de la organización, y para la generación de conocimiento pertinente a las necesidades del país. Reiterando un concepto enunciado anteriormente, en el caso de las entidades estatales, y siendo el empleador el mismo Estado de Chile la integración es obvia y evidente en cargos mixtos o complementarios. Esta visión no es aplicable solo a las instituciones estatales, sino que también a instituciones privadas, como lo ha demostrado el vínculo de la Facultad de Medicina de la Universidad Católica de Chile con el Hospital Dr. Sótero del Río, o de la Universidad de Concepción o la Universidad Austral de Chile con los hospitales respectivos.

Es evidente que el desarrollo global del sistema universitario nacional es insuficiente para poblar con este modelo todos los hospitales y otros recintos asistenciales públicos del país. Pocas universidades tienen la capacidad de satisfacer las condiciones enunciadas antes; un número importante de carreras de la salud funciona sobre la base de la contratación a honorarios de servicios de profesionales como docentes, la minoría cuenta con departamentos universitarios asociados a servicios clínicos, pocas hacen investigación. Estas insuficiencias actuales, así como la dificultad de la tarea de revertir una situación notablemente alejada de la búsqueda de la calidad y la defensa implícita de los intereses que la sustentan, no pueden ser excusas para no construir un mejor sistema. Consideramos totalmente factible proyectar un desarrollo de hospitales de alta calidad asistencial y docente a partir de las situaciones actuales, que sea gradual, sostenido y programado en los plazos compatibles con la construcción de la calidad de sistemas complejos; el determinante fundamental es la voluntad de articular recursos ya existentes de manera inteligente y en función del interés común.

Chile consiguió excelencia en educación en salud cuando era un país de mucho menor desarrollo, gracias a la visión de las universidades y gobiernos de sus épocas; no hay razón para que eludamos la responsabilidad de proyectarlo al futuro, sobre la base de la evidencia, el resguardo del interés público y la mirada de largo plazo que el bienestar de nuestra población requiere.

\section{Referencias}

1 http://www.usnews.com/info/blogs/press-room/articles/2016-08-02/us-news-announces-the-201617-besthospitals

2 http://health.usnews.com/best-hospitals/rankings

3 http://www.abcnewspoint.com/top-10-best-hospitalsworld/ 